\title{
Double Exchange Model for Magnetic Hexaborides
}

\author{
Vitor M. Pereira, ${ }^{1,2}$ J. M. B. Lopes dos Santos, ${ }^{2}$ Eduardo V. Castro, ${ }^{2}$ and A. H. Castro Neto ${ }^{1}$ \\ ${ }^{1}$ Department of Physics, Boston University, 590 Commonwealth Avenue, Boston, Massachusetts 02215, USA \\ ${ }^{2}$ CFP and Departamento de Física, Faculdade de Ciências Universidade do Porto 4169-007 Porto, Portugal.
}

(Dated: 29th June 2018)

\begin{abstract}
A microscopic theory for rare-earth ferromagnetic hexaborides, such as $\mathrm{Eu}_{1-x} \mathrm{Ca}_{x} \mathrm{~B}_{6}$, is proposed on the basis of the double-exchange Hamiltonian. In these systems, the reduced carrier concentrations place the Fermi level near the mobility edge, introduced in the spectral density by the disordered spin background. We show that the transport properties such as Hall effect, magnetoresitance, frequency dependent conductivity, and DC resistivity can be quantitatively described within the model. We also make specific predictions for the behavior of the Curie temperature, $T_{C}$, as a function of the plasma frequency, $\omega_{p}$.
\end{abstract}

PACS numbers: 71.23.An, 75.47.Gk, 75.47.-m

The series of compounds $\mathrm{R}_{1-x} \mathrm{~A}_{x} \mathrm{~B}_{6}$, where $\mathrm{A}$ is an alkaline-earth metal such as $\mathrm{Ca}$ or $\mathrm{Sr}$, and $\mathrm{R}$ a rareearth magnetic ion, has recently attracted considerable interest, following a series of experiments which unveiled intriguing connections between their magnetic, transport and optical properties. These are cubic compounds where a divalent [1] lanthanoid occupies the central position on a cube, surrounded by eight $\mathrm{B}_{6}$ octahedra at each vertex. Boron atoms make up a rigid cage, held together by covalent bonds between neighboring $\mathrm{B}$ atoms. Our focus will be on the results known for $\mathrm{Eu}_{1-x} \mathrm{Ca}_{x} \mathrm{~B}_{6}$. $\mathrm{EuB}_{6}$ is a ferromagnetic metal, ordering at $T_{C} \approx 15 \mathrm{~K}$, and characterized by a quite small effective carrier density, of order of $10^{-3}$ per unit cell, at high temperatures [2, 3, 4]. Magnetism is found to arise from the half-filled $4 f$ shell of $\mathrm{Eu}$, whose localized electrons account for the measured magnetic moment of $7 \mu_{\mathrm{B}}$ per formula unit $[3,4,5]$. The FM transition temperature is reported to decrease with increasing Ca content [4, 6] and the totally substituted compound $\mathrm{CaB}_{6}$ exhibits no magnetism.

In this paper we propose a simple model that describes quantitatively the properties revealed by the experiments done in $\mathrm{EuB}_{6}$ : (i) a precipitous drop in the DC resistivity just below $T_{C}$, with a change by a factor as high as 50 between $T_{C}$ and the lowest temperatures [3, 4]; (ii) the large negative magnetoresistance observed near $T_{C}$; (iii) an increase in the number of carriers, by a factor of $2-3$, upon entering the ordered phase, as evidenced by Hall effect [4]; (iv) a large blue shift of the plasma edge, seen also for $T \leq T_{C}$, both in reflectivity, $R(\omega)$, and polar Kerr rotation [2, 7, 8]; (v) a scaling of the plasma frequency with the magnetization. The above listed features constitute a case for the strong coupling of the magnetization to the transport properties.

The effects of chemical doping with non-magnetic $\mathrm{Ca}$ are also considered, and the theory explains qualitatively the following experimental findings: (1) with doping, $x$, the metallic regime, found in $\mathrm{EuB}_{6}(x=0)$, evolves to a semiconducting behavior above $T_{C}[4,6]$; (2) just below $T_{C}$ the carrier density increases by at least two orders of magnitude [4]; (3) the plasma edge is visibly smeared while the corresponding resonance in the polar Kerr rotation is greatly attenuated [9, 10]; $(4) \rho(T, H)$ and $\omega_{p}$ display an exponential dependence in the magnetization 6, 11]; (5) there remains a significant and rapid decrease of $\rho(T)$ just below $T_{C}$, albeit by a smaller factor than in the undoped case. Our model predicts that the square of the plasma frequency, $\omega_{p}^{2}$, scales linearly with the Curie temperature, $T_{C}$, as doping $x$ is varied. Furthermore, we also present a phase diagram $T$ versus $x$ which contains two phase transition lines: paramagnetic to ferromagnetic and metal to insulator.

Theoretical understanding of the microscopic mechanisms responsible for the transport data is still controversial. Earlier local density approximation (LDA) calculations predicted semi-metallic character with an overlap of conduction and valence bands at the Fermi level on the $X$ point of the Brillouin zone 12, 13]. This appears to be consistent with the Shubnikov-de Haas and de Haas-van Alphen measurements [14, 15]. Further density functional theory (DFT) calculations showed the existence of a sizeable gap of $\sim 1 \mathrm{eV}$. These results are corroborated by angle resolved photoemission spectroscopy (ARPES) for magnetic [16] and non-magnetic hexaborides [17]. Some existing theoretical models assume the semimetal scenario [11, 18, 19], that ARPES seems to rule out.

We present a microscopic theory for $\mathrm{EuB}_{6}$, involving a single conduction band, consistent with the observed properties of these compounds, and make specific predictions regarding the phase diagram for $\mathrm{Eu}_{1-x} \mathrm{Ca}_{x} \mathrm{~B}_{6}$. The presence of defects in the crystalline environment 16, 20, 21] is believed to create a small carrier concentration in the conduction band. These carriers interact with the local Eu magnetic moments in a cubic lattice, via an $s-f$ exchange coupling, leading to the well known Kondo lattice Hamiltonian:

$$
\mathcal{H}_{K L}=-\sum_{\langle i, j\rangle \sigma}\left(t_{i, j} c_{i, \sigma}^{\dagger} c_{j, \sigma}+\text { h.c. }\right)+J \sum_{i} \overrightarrow{\mathbf{s}}_{i} \cdot \overrightarrow{\mathbf{s}}_{i}
$$


where $\overrightarrow{\mathbf{S}}_{i}$ represents the $4 f$ Eu spin $(S=7 / 2)$ operator at the site $i$ and $\overrightarrow{\mathbf{s}}_{i}=1 / 2 \sum_{\alpha, \beta} c_{i, \alpha}^{\dagger} \vec{\tau}_{\alpha, \beta} c_{i, \beta}$ is the the electron spin operator. Given the large value of $S$, the core spins are treated as classical variables parameterized by the polar and azimuthal angles as $\overrightarrow{\mathbf{S}}_{i}=$ $S\left(\sin \theta_{i} \cos \phi_{i}, \sin \theta_{i} \sin \phi_{i}, \cos \theta_{i}\right)$. Based on the current literature, reasonable ranges for the model parameters are $t_{i j}=t \sim 0.1-1 \mathrm{eV}$ and $J S \sim 0.35-0.7 \mathrm{eV}$.

The carrier concentration in $\operatorname{EuB}_{6}\left(n \sim 10^{-3}\right)$ is very small, and therefore the mean kinetic energy is much smaller than the magnetic one, $\langle K\rangle \sim t n \ll J S$. In this regime, the low energy physics of Hamiltonian (11) can be obtained by taking the limit of $J \rightarrow \infty$ [22]. The effective Hamiltonian is obtained by projecting out the states with the electronic spin parallel (for $J>0$ ) or antiparallel (for $J<0$ ) to the local core spin $\overrightarrow{\mathbf{S}}_{i}$. In either case, the result is nothing more than the double-exchange (DE) Hamiltonian 23]:

$$
\mathcal{H}=-\sum_{\langle i, j\rangle}\left(\widetilde{t}_{i, j} c^{\dagger} c_{j}+\text { h. c. }\right)
$$

The effective hoping amplitude is $\tilde{t}_{i, j}=$ $t \cos \left(\theta_{i} / 2\right) \cos \left(\theta_{j} / 2\right)+\sin \left(\theta_{i} / 2\right) \sin \left(\theta_{j} / 2\right) e^{-i\left(\phi_{i}-\phi_{j}\right)}$.

The Hamiltonian (2) describes a very interesting interplay between the magnetic and electronic degrees of freedom. The spin texture acts as a static disordered background for the electronic motion [24]. The ground state of the problem is obtained by minimizing the kinetic energy with the alignment of all the spins in the system. Thus, the electronic density of state (DOS), $N(E, M)$, and the Fermi energy, $E_{F}(M)$, change with magnetization, $M$, even if the number of electrons is constant. In this ferromagnetic state the transport properties are tied to the magnetic ones. Similar physics can be found in the context of the colossal magnetoresistance (CMR) manganites [25]. This is indeed an intrinsically disordered problem where the strength of the non-diagonal disorder is determined by $M$. A mobility edge, $E_{C}(M)$, appears in the spectral density [26] and strongly depends on $M$. In the absence of structural disorder, when the system is fully magnetized $(M=1)$ the mobility edge moves out of the band. One immediately realizes that in the paramagnetic phase $(M=0)$, if $E_{C}(0)$ and $E_{F}(M=0)$ are comparable, the onset of ferromagnetism at $T_{C}$ and the concomitant displacement of $E_{C}$ towards the bottom of the band, have a major effect in the transport properties, by allowing more states to become delocalized and consequently increasing the conductivity. This was actually a concept that lingered for some time in the context of the manganites where the metal-insulator transition and CMR were assumed to stem from this effect [27]. In that case spin disorder alone cannot account for the experimental evidence since $E_{C}(0)$ encloses less than $0.5 \%$ of the states 28]. In the case of $\mathrm{EuB}_{6}$, however, the small number of localized states is comparable to the concen-

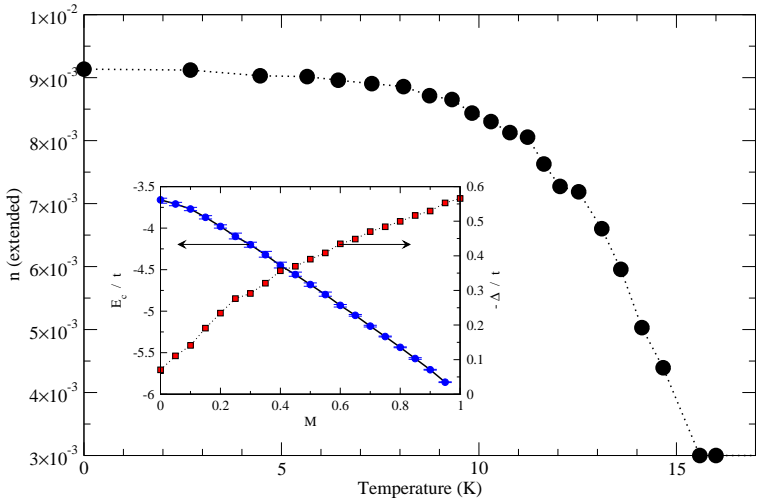

Figure 1: Evolution of $n_{e}(T)$ assuming $n_{e}^{0}=0.003$ and $E_{W}=0.1 t$. The inset shows the mobility edge $\widetilde{E}_{C}$ (circles) and mobility gap $\Delta$ (squares) as functions of the normalized magnetization $M$.

tration of mobile carriers and thus this effect should have visible consequences.

"Pure" $E u B_{6}$. In order to investigate the aforementioned, we calculate $N(E, M)$ and $E_{C}(M)$ for the Hamiltonian (2) for static spin configurations with specific values of $M$. The full, self-consistent treatment of the magnetism of Hamiltonian (2) is computationally too demanding for the system sizes required to study localization effects. In this work we circumvent these difficulties by assuming uncorrelated spins in an external field and extracting the temperature dependence of the zero-field magnetization, $M(T)$, from the experimental data [5]. The recursion method [29] was used to calculate $N(E, M)$, and $E_{C}(M)$ was located using the transfer matrix method 30, 31]. Non-magnetic defects were accounted by a small magnetization independent shift, $E_{W}$, of $E_{C}$, thus giving effectively $\widetilde{E}_{C}(M)=$ $E_{W}+E_{C}(M)$. The important quantity is the mobility gap, $\Delta(M)=\widetilde{E}_{C}(M)-E_{F}(M)<0$, the variation of which, at the transition, completely determines the number of extended (metallic) carriers, $n_{e}(M)$. Tuning $E_{F}(0)$ so that $n_{e}^{0} \equiv n_{e}(0)=0.003$, as reported in the transport measurements above $T_{C}$, the variation of $n_{e}$ is presented in Fig. 1. $\Delta(M)$ is found to be almost linear in $M: \Delta(M)=\Delta_{0}(1-\alpha M)$ (see the inset in Fig. 11). The range of variation of $n_{e}$ in Fig. [1 compares well with the data obtained from the Hall effect by Paschen et al. [4].

Another experimentally accessible quantity is the plasma frequency, $\omega_{p}$. Applying the Kubo formula for the optical conductivity, $\sigma(\omega)$, to Hamiltonian (2) the sum rule for its real part, $\sigma^{\prime}(\omega)$, can be extracted 32] and combined with the optical sum rule [33] leading to the plasma frequency for the model $\omega_{p}^{2}=-4 \pi e^{2} a^{2}\langle\mathcal{H}\rangle / 3$.

In the reported experimental results [7], $\omega_{p}^{2}$ is obtained from the Drude contribution to the optical response of the material. Therefore, in order to compare with the 


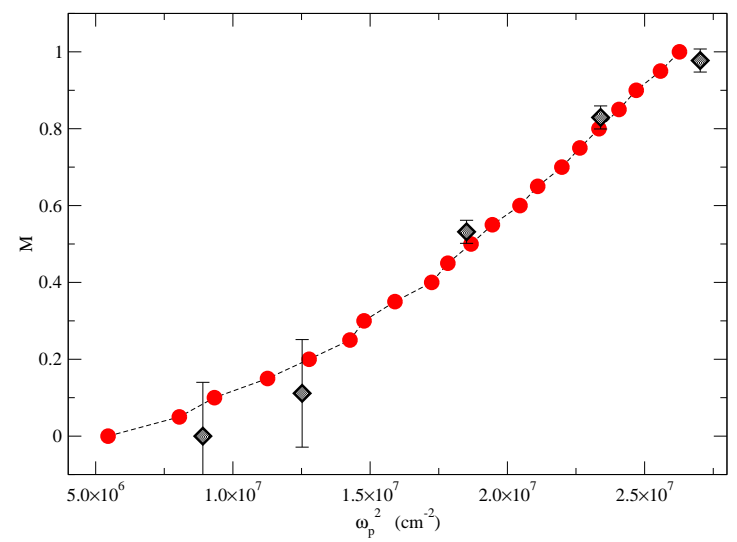

Figure 2: Result for $\omega_{p}^{2}(M)$ obtained from the sum rule (circles) and experimental data (diamonds). The experimental $(H=0)$ points were obtained combining $\omega_{p}^{2}(T)$ at zero field from ref. [7] with the zero field $M(T)$ from ref. [5].

model predictions, we included only the contribution of metallic states in the calculation of the kinetic energy, $\omega_{p}^{2}(M) \sim \int_{E c(M)}^{\infty} N(E, M) f(E) E d E$. The outcome of such procedure being shown in Fig. 2 The linear relation $\omega_{p}^{2} \sim M$ inferred from ref. [34], for high $M$, is indeed verified. These results for $\omega_{p}$ are obtained with $t=0.55 \mathrm{eV}$.

The steep drop in the resistivity below $T_{C}$ can be understood in a consistent way: the resistivity is dominated by spin and phonon scattering at high temperatures until the magnetic transition is reached. Below this point the negative mobility gap increases along with $M$, delocalizing a considerable amount of the previously localized states. The scaling theory of localization [35] prescribes that $\sigma(M) \sim(-\Delta(M))^{\nu}$. Replacing here the results for

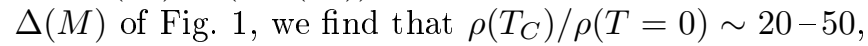
in agreement with the values obtained in the experiments $[3,4]$. The negative magnetoresistance (MR) comes about as a natural consequence of our microscopic mechanism and further supports our claim that the dependence of the mobility gap on the magnetization is the most relevant factor driving the physics of this material near $T_{C}$.

Doped $E u B_{6}$. Mean-field analysis of Hamiltonian (2) predicts that $T_{C}$ scales with $\langle\mathcal{H}\rangle[36,37]$. Since $\omega_{p}^{2}$ follows the same scaling, as stated above, we expect that in the series $\mathrm{Eu}_{1-x} \mathrm{Ca}_{x} \mathrm{~B}_{6}$ the squared plasma frequency should scale approximately with $T_{C}$, a prediction that would be interesting to investigate experimentally with additional infrared reflectivity experiments.

Band structure calculations seem to agree that the conduction band has a strong $5 d$ Eu component. Ca doping not only dilutes the magnetic system but also the conducting lattice. The hoping parameter $\widetilde{t}_{i, j}$ in eq. (2) is then replaced by $\widetilde{t}_{i, j} p_{i} p_{j}$, where $p_{i}=1$ if the site $i$ is oc- cupied by a Eu atom and $p_{i}=0$, otherwise. The microscopic problem thus becomes a DE problem in a percolating lattice which, at $T=0 \mathrm{~K}$, reduces to a quantum percolation problem [38]. Since $\mathrm{Ca}$ and $\mathrm{Eu}$ are isovalent in hexaborides one does not expect the number of carriers to depend on $x$. Nevertheless, since carriers are presumed to arise from defects it is difficult to be specific on this issue. The mobility edge, on the other hand, is very sensitive to the $\mathrm{Eu} \rightarrow$ Ca substitution, and should drift toward the band center. In the paramagnetic regime $\left(T>T_{C}\right), \widetilde{E}_{C}$ should move past the Fermi energy at some critical doping $x_{M I}^{P}$, after which the mobility gap becomes positive. This determines a crossover from the metallic regime to an insulating behavior for $T>T_{C}$, as seen in the doped compounds [4, 6]. At finite $T$ the mobile carriers arise from thermal activation across the mobility gap. The resistivity should display a semiconducting behavior with $T$ and its dependence on $M$ should be dominated by an exponential factor $\rho(M) \sim \exp \left(\Delta(M) /\left(k_{B} T\right)\right)$ [39]. Using $\Delta(M) \approx \Delta_{0}(1-\alpha M)$, as happens in the non-diluted case for either of the $\Delta(M) \lessgtr 0$ situations, we find that $\rho(M) \sim \exp (-\beta M)(\beta$ is a constant $)$, as seen in the experiments [6].

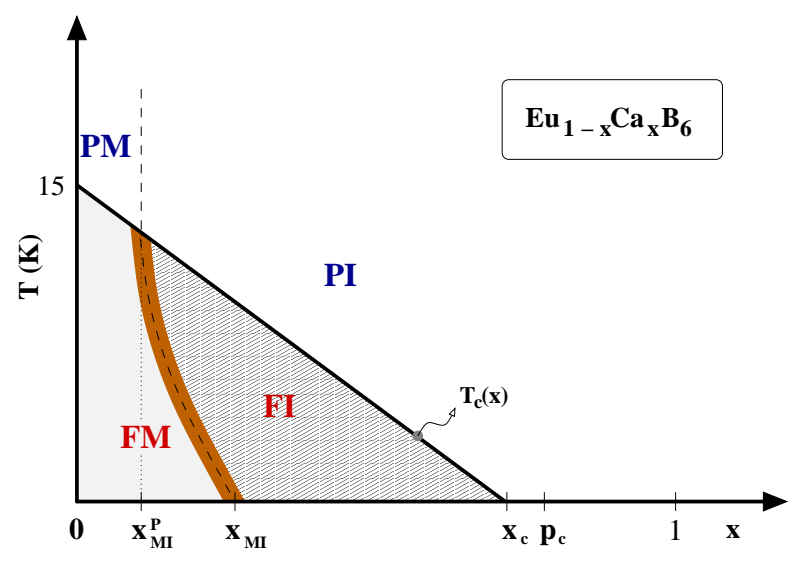

Figure 3: Schematic phase diagram for $\mathrm{Eu}_{1-x} \mathrm{Ca}_{x} \mathrm{~B}_{6}$ in the $T-x$ plane in standard notation: PM stands for paramagnetic metal, PI for paramagnetic insulator, FM means ferromagnetic metal and FI ferromagnetic insulator.

As $T$ is lowered below $T_{C}$ and $M$ increases on the percolating cluster, one expects the crossover from metallic to semiconducting behavior to occur at larger values of $x$; this is illustrated in Fig. [3] by the curved dashed line separating the FM and FI regions. In the vicinity of this line, a sharp metal-semiconducting distinction is not possible, resulting possibly in a bad-metal behavior. At $T=0$, there is a metal-insulator transition occurring at a concentration $x_{M I} \geq x_{M I}^{P}$, which corresponds to the quantum percolation transition for a small number of carriers (this is different from the usual quantum percolation point $p_{Q}<p_{c}$, which is defined by the lo- 
calization of all states in the band [40]). Even though we expect $x_{M I}$ to be close to $x_{M I}^{P}$, the possibility of a semiconducting behavior crossing over to metallic at low $T$ (for some $x_{M I}^{P}<x<x_{M I}$ ) cannot be excluded. Ferromagnetism induced by the DE mechanism is expected to persist past $x_{M I}$ as long as the localization length is greater than the lattice spacing. Naturally, the critical concentration, $x_{c}$, where $T_{C} \rightarrow 0$, should not be higher than $p_{c} \approx 0.69$, the classical site-percolation threshold for the simple cubic lattice [41]. The values of $x_{M I}^{P}$ and $x_{M I}$ in Fig. 3 vary with carrier density and are expected to be sample dependent, since carriers seem to originate from defects. In fact, annealing experiments can be quite important for the study of the phase diagram.

Some questions remain as yet unanswered. The value of $t=0.55 \mathrm{eV}$ used in Fig 2 leads to a mean-field estimate of $T_{C} \approx 80 \mathrm{~K}[37$ ] which is much higher than the experimental value. At such low carrier concentrations, magnetic polaron formation [19, 42] must certainly be taken into account close to $T_{C}$ and could lead to a reduction of the mean-field estimate. Indeed, Raman scattering studies [43, 44] close $T_{C}$ have been interpreted in terms of magnetic polarons. A large, positive, $\mathrm{MR}$ is observed at high fields and $T<T_{C}$ in $\mathrm{EuB}_{6}[4$, 14]. If $J>0$, it is possible that high magnetic fields may bring into play states of parallel electronic and Eu spins, that were projected out to obtain the Hamiltonian (2). One would then have to deal with two types of carriers and therefore a positive MR. Finally, our model allows for the unusual situation of a competition between conduction by thermal activation across a small, magnetization dependent, mobility gap, and conduction by hopping among localized states. Some of the details of the transport properties, such as the remarkable scaling of the Hall coefficient with magnetization in $\mathrm{Eu}_{0.6} \mathrm{Ca}_{0.4} \mathrm{~B}_{6}$ [6], will require a more thorough understanding of transport in these circumstances.

In conclusion, we have proposed a theory for Eu hexaborides, based on the close proximity of the Fermi level and a magnetization dependent mobility edge $E_{C}(M)$. This theory arises quite naturally from the Kondo lattice problem in the limit of very small number of carriers; it accounts, in a quantitative way, for many of the observed experimental properties in these materials. Furthermore, we also predict a linear scaling of $\omega_{p}^{2}$ with $T_{C}$ that can be tested in infrared reflectivity experiments.

We thank L. Degiorgi, P. Littlewood, H. Ott, and C. Varma for illuminating conversations. V.M.P., E.V.C. and J.M.B.L.S. are financed by FCT and the European Union, through POCTI(QCA III). V.M.P. further acknowledges Boston University for the hospitality and the financial support of F.C.T., through grant ref. SFRH $/$ BD $/ 4655 / 2001$. A.H.C.N. was partially sup- ported through NSF grant DMR-0343790.

[1] Z. Fisk et al., J. Appl. Phys. 50, 1911 (1979).

[2] L. Degiorgi et al., Phys. Rev. Lett. 79, 5134 (1997).

[3] S. Süllow et al., Phys. Rev. B 57, 5860 (1998).

[4] S. Paschen et al., Phys. Rev. B 61, 4174 (2000).

[5] W. Henggeler et al., Sol. State Comm. 108, 929 (1998).

[6] G. A. Wigger et al., Phys. Rev. B 66, 212410 (2002).

[7] S. Broderick et al., Phys. Rev. B 65, 121102(R) (2002).

[8] S. Broderick et al., Eur. Phys. J. B 33, 47 (2003).

[9] A. Perucchi et al., Phys. Rev. Lett. 92, 67401 (2003).

[10] G. Caimi et al., Phys. Rev. B 69, 12406 (2003).

[11] G.A. Wigger et al., cond-mat/0309412 (2003).

[12] S. Massidda et al., Z. Phys. B. 102, 83 (1996).

[13] C. O. Rodriguez et al., Phys. Rev. Lett. 84, 3903 (2000).

[14] M. C. Aronson et al., Phys. Rev. B 59, 4720 (1999).

[15] It should be stressed that this experiments require large magnetic fields.

[16] J. D. Denlinger et al., Phys. Rev. Lett. 89, 157601 (2002).

[17] S. Souma et al., Phys. Rev. Lett. 90, 27202 (2003).

[18] J. C. Cooley et al., Phys. Rev. B 56, 14541 (1997).

[19] M.J. Calderón et al., cond-mat/0312437 (2003).

[20] M. A. Noack et al., J. of Cryst. Growth 49, 595 (1980).

[21] R. Monnier et al., Phys. Rev. Lett. 87, 157204 (2001).

[22] V. M. Pereira et al, unpublished (2003).

[23] P. W. Anderson and H. Hasegawa, Phys. Rev. 100, 675 (1955).

[24] The is a valid approximation in the large- $S$ limit.

[25] E. Dagotto, Nanoscale Phase Separation and Colossal Magnetoresistance (Springer Verlag, 2003).

[26] E. N. Economou and M. H. Cohen, Phys. Rev. B 5, 2931 (1972).

[27] C. M. Varma, Phys. Rev. B 54, 7328 (1996).

[28] Q. Li et al., Phys. Rev. B 56, 4541 (1997).

[29] R. Haydock, in Solid State Physics (Academic Press, New York, 1980), vol. 35, pp. 216-294.

[30] A. MacKinnon and B. Kramer, Phys. Rev. Lett. 47, 1546 (1981).

[31] J. L. Pichard and G. Sarma, J. Phys. C 14, L127 (1981).

[32] P. F. Maldague, Phys. Rev. B 16, 2737 (1977).

[33] F. Wooten, Optical Properties of Solids (Academic Press, New York, 1972).

[34] S. Broderick et al., Eur. Phys. J. B 27, 3 (2002).

[35] P. A. Lee and T. V. Ramakrishnan, Rev. Mod. Phys. 57, 287 (1985).

[36] P.-G. de Gennes, Phys. Rev. 118, 141 (1960).

[37] K. Kubo and N. Ohata, J. Phys. Soc. Jpn. 33, 21 (1972).

[38] Yonathan Shapir et al., Phys. Rev. Lett. 49, 486 (1982).

[39] N. F. Mott and E. A. Davis, Electronic processes in noncrystalline materials (Clarendon Press, Oxford, 1971).

[40] I. Chang et al., Phys. Rev. Lett. 74, 2094 (1994).

[41] D. Stauffer and A. Aharony, Introduction to Percolation Theory (Taylor \& Francis, London, 1994), 2nd ed.

[42] L. G. L. Wegener and P. B. Littlewood, Phys. Rev. B 66, 224402 (2002).

[43] P. Nyhus et al., Phys. Rev. B 56, 2717 (1997).

[44] C. S. Snow et al., Phys. Rev. B 64, 174412 (2001). 